\title{
New Hybrid System of Swarm Intelligence using for Minimal Path Travel on Contours
}

\author{
Matej Babič ${ }^{1, *}$ \\ Faculty of Information studies, Novo Mesto, Slovenia
}

\begin{abstract}
Anyone who observes ant colonies and swarms of bees gets the impression that they are following a certain plan. They are small states in which everyone seems to know their job and the sequence of which has always fascinated people: How would it be if this principle could be transferred to companies? In this article is presented new hybrid system of swarm intelligence using for minimal path travel on contours in mechanical engineering, especially in laser technology of hardening.
\end{abstract}

Keywords: Hybrid system, Swarm intelligence, Ant colony, Bee colony

\section{Introduction}

With the widespread popularity and application of machine learning, especially deep learning, as an important tool for solving such problems, optimization problems and methods are becoming more and more familiar to everyone. Even in the field of machine learning, it can be roughly considered that all machine learning problems can finally be transformed into optimization problems. The general formulation of the optimization problem in engineering design is to select a set of parameters (variables) to make the design index (target) reach the optimal value under the condition that a series of related constraints are met. Therefore, the optimization problem can usually be expressed as a problem in the form of mathematical programming [1].

Swarm intelligence is a sub-area of artificial intelligence, which is about the fact that a number of simple elements (particles, agents, objects, etc.) only interact with one another on the basis of simple, local rules $[2,3]$. There is no central regulation. However, if the local rules are designed appropriately, a superordinate structure with self-organized, collective intelligence results. Typical examples are ant colonies, bee colonies, schools of birds and fish. Without the individual being aware of the overall situation or only following very simple local rules, an overall system with astonishing properties and functionalities still results. Technical application examples can be found in robotics and in the solution approach for "intelligent" traffic control [4]. So, swarm intelligence, as an emerging field, has become the hot spot and frontier field of artificial intelligence as well as economic, social, biological, engineering and other interdisciplinary fields.

Laser hardening is a heat treatment technology that uses a laser to heat the surface of a material above the phase transition point [5]. As the material cools, austenite is transformed into martensite, thereby hardening the surface of the material. The local quenching deformation produced by the laser quenching process when quenching the machined components is very small, which can reduce the subsequent processing to a minimum or completely eliminate this processing, so that the mold can be hardened after quenching. The laser beam quenching equipment can be extended to laser powder surfacing and laser welding wire surfacing. Workpiece materials that 
can be hardened include structural steel, quenched and tempered steel and cast steel, as well as various types of cast iron such as flake graphite cast iron or nodular graphite cast iron. The material that can be hardened directly has a carbon content of at least $0.22 \%$. However, carburized steel and pre-nitrided steel can also be hardened [6].

The mold surface is heated to the austenitizing temperature by a temperature rise rate greater than $1000 \mathrm{~K} / \mathrm{s}$. This temperature rise is controlled to be close to the melting point of the material, but will not reach this melting temperature. The temperature can be maintained for about $10^{-3}$ to 10 seconds, and the cooling rate is determined by the temperature conduction to the component itself. A thin oxide layer is formed on the surface of the component. This oxide layer is mostly insignificant and can be easily removed. In the case of gas protection, the formation of oxide layers can be avoided during laser quenching. Compared with other quenching processes, the metallographic structure obtained by laser hardening is quite fine particles [7].

If the heat transferred into the workpiece during quenching is high, the stress formed by the previous cutting process will be released around the workpiece, causing deformation of the component. The workpiece needs to be left with a machining allowance, which requires a lot of material costs and a certain risk. As a result, subsequent processing must be performed on the hardened components, which will consume more time and expense. As the first lecture of this series, this article will briefly explain some specific examples of constructing the objective function, as well as incorporating some common algorithms into the optimization system. The goal is to make the component in the "soft" state, that is, to perform cutting and finishing before quenching, which requires a quenching process that produces little deformation. The use of lasers for local quenching provides the best conditions for reducing quenching deformation. And can continue to maintain the basic toughness of the component core and correspondingly reduce the risk of cracks.

The aim of this research is present a new hybrid system of swarm intelligence using for minimal path travel on contours in mechanical engineering, especially in laser technology of hardening. It means that we combine two different method of swarm intelligence into one and use it in mechanical engineering.

\section{Methodology}

I used two methods of swarm intelligence, namely ant colony and bee colony using for minimal path travel on contours. The traveler can create an optimal sequence of stops along a route. This is called the traveling salesman problem (TSP). The TSP is a combinatorial problem, that is, there is no direct method for determining the optimal sequence. To quickly find the suitable solutions for problems of this kind, heuristic algorithms are used. The implementation of TSP in minimal path travel

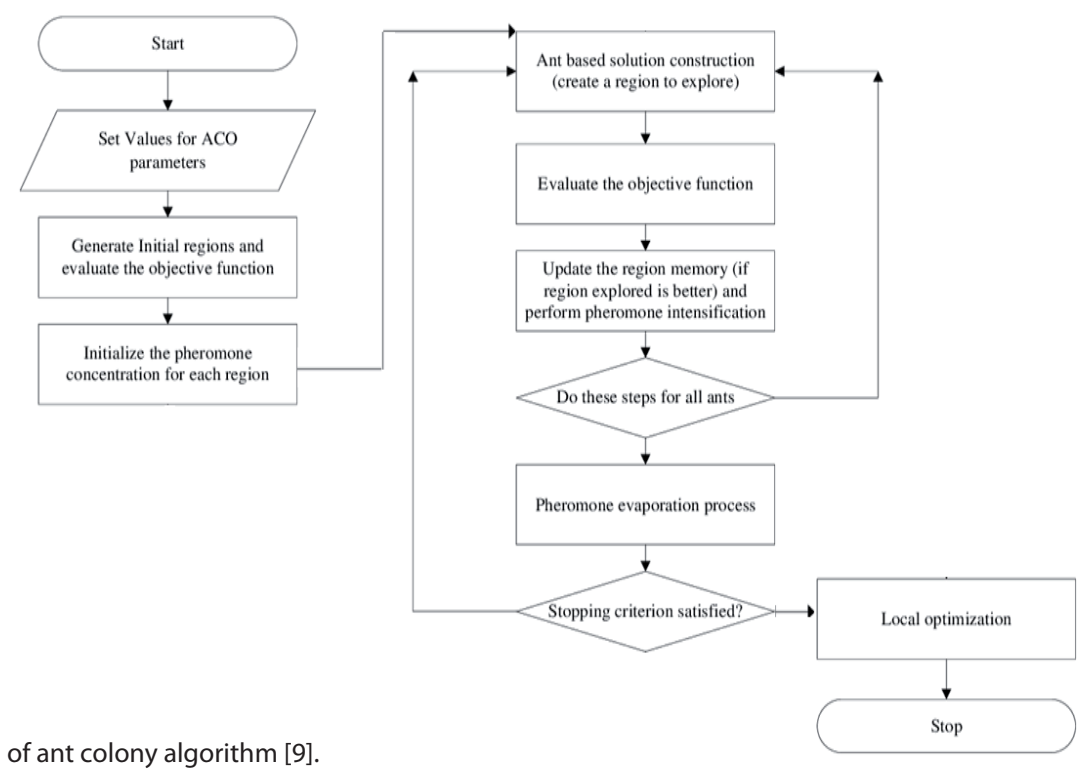

Figure 1: Diagram of ant colony algorithm [9]. 
also allows you to manage time windows at stops; this means that it searches for the optimal sequence of visiting stops with a minimum delay.

A colony finds solutions to problems that would be unthinkable for individual ants [8]. The colony uses the shortest route to the best source of feed, distributes tasks to the workers and defends the territory. Ants are helpless as individuals, but as a colony they respond quickly and efficiently to their environment. The ability to do this is called collective intelligence or swarm intelligence. On this basis, computer scientist Marco Dorigo from the Free University of Brussels developed the first mathematical methods for solving complicated problems for people back in 1991: route planning for trucks, flight schedules for airlines, or the control of military robots [9]. The general algorithm is relatively simple and based on a set of ants, each making one of the possible round-trips along the cities. Figure 1 represent diagram of ant colony algorithm.

Colonies of honey bees have been of interest to mankind for many years, these creatures are recognized as one of the most amazing creations of wildlife [10]. A clear organization of the life of bees in the colonies, the correct geometry of honeycombs, the cultivation of millions of offspring and hundreds of other factors have led to the beginning of modern scientific research that gives us an understanding of one of the most unusual structures. Studying the processes of the brain and the visual functions of these insects makes it possible to create new technical devices and introduce modern technologies, such as Face-ID, using the amazing properties of bees in their modeling, which is certainly relevant for modern society. Figure 2 presents diagram of bee colony algorithm.

Bellow is presented new developed hybrid algorithm ant colony and bee colony.

At each stage, the ant/bee chooses to move from one point on surface to another according to some rules:

- It must visit each point on surface exactly once;

- Each employed ant/bee goes to a food source in her memory and determines a closest source, then evaluates its nectar amount and dances in the hive

- Each onlooker watches the dance of employed ant/bees and chooses one of their sources depending on the dances, and then goes to that source. After choosing a neighbor around that, she evaluates its nectar amount.

- A distant point on surface has less chance of being chosen (the

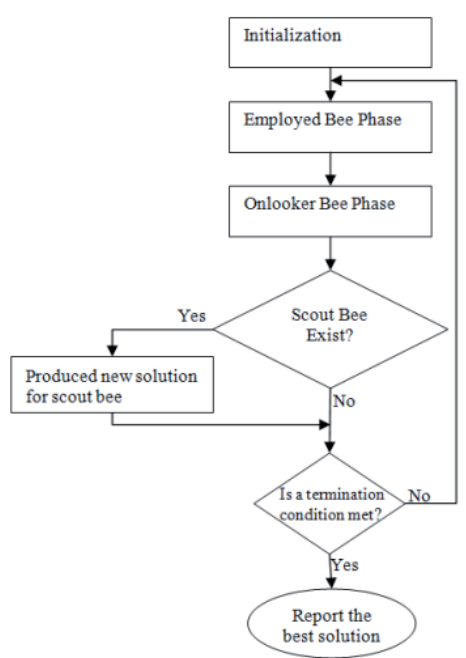

Figure 2: Diagram of bee colony algorithm [11].

visibility);

- The more intense the pheromone trail laid out on an edge between two points on surface, the greater the probability that that edge will be chosen;

- Having completed its journey, the ant/bee deposits more pheromones/trail path on all edges it traversed, if the journey is short; - After each iteration, trails of pheromones/trail path evaporate.

- Abandoned food sources are determined and are replaced with the new food sources discovered by scouts.

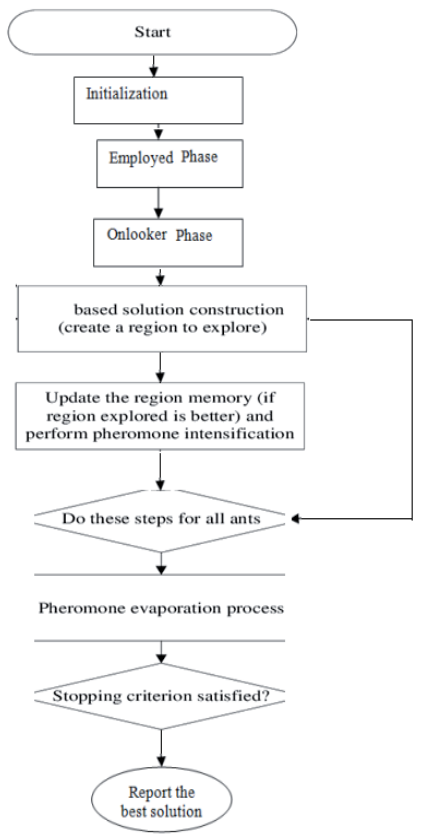

Figure 3: Diagram of hybrid algorithm ant colony and bee colony. 


\section{Experimental work and material preparation}

A robot laser cell RV60-40 (Reis Robotics Company) was used for hardening. RV60-40 is vertical robot with 6 free programmable axis application in full or semi automatic production areas. This robot laser cell can work on parts loaded and unloaded using a rotary indexing table. This cell is highly flexible and can be easily configured to suit a variety of laser material processing applications (laser hardening and laser welding). It is very useful for heat treatment of complex 3D structures with complex contours. Fig. 4 represent Robot laser cell for hardening. Algorithm was implemented as computer program. The software was used from Robot Reis (Reis Robotics Company). The algorithm was introduced to the software from computer.

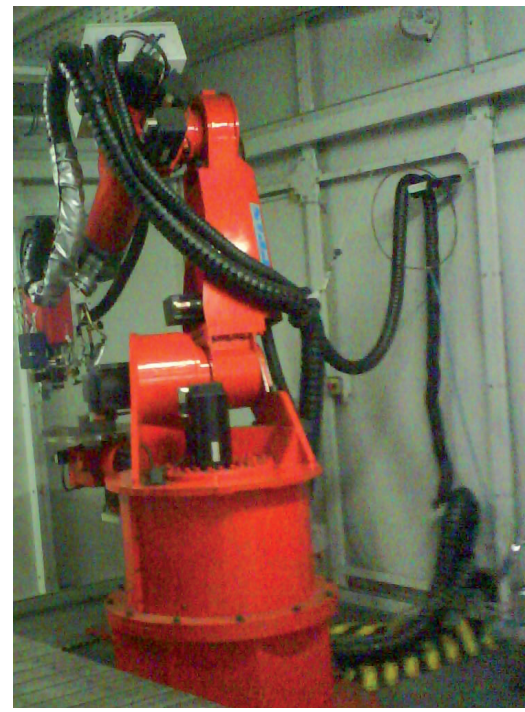

Figure 4: Robot laser cell for hardening.

On Fig. 4 is presented one example of 3D complex surface tool steel from automobile industry.

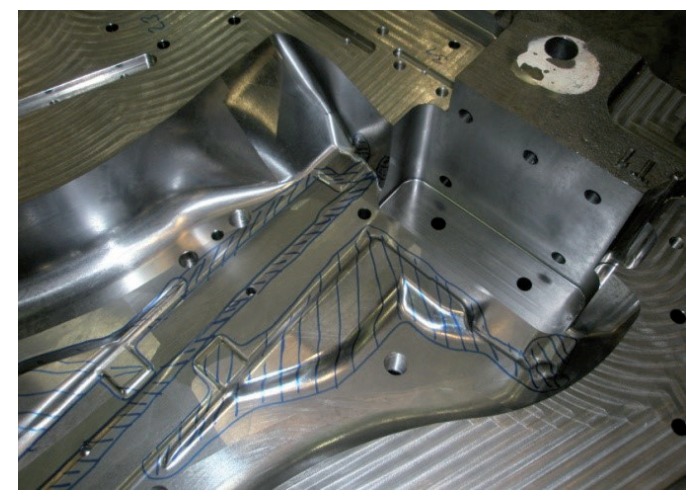

Figure 5: Example of 3D complex surface tool steel from automobile industry.

\section{Results}

In the automotive industry, 3D complex metal structures must be well reinforced, have good wear resistance and long service life. Thus, I present one example using laser technique of hardening in 3D complex structure, by using swarm intelligence.

On Fig. 6 is represented 3D complex tool steel with different trajectory of surface and optimal way to hardened tool steel. Also, Fig. 6 represent different trajectory computed with hybrid algorithm. Fig. 6 represent a)-c) examples of initial trajectories and Fig. 6 d) represent final trajectory. Open problem: How to find minimal path of hardened area of complex 3D surface tool steel? In the article hybrid algorithm bee and ant colony was adapt to find optimal path of hardened area of complex 3D surface tool steel. On Fig. 6 it can be seen that first path of laser beam. In this position laser beam need maximum of time and energy. In the second step (Fig. 6b), can be seen that the laser beam finds short path like in first position. In the third step (Fig. 6c), can be seen that the laser beam approximates very close to the optimal path. In the last step (Fig. $6 \mathrm{~d}$ ), can be seen that the laser beam finds the optimal path and need minimal time and energy. In first step, the laser beam travel 25 time from point to point. In second step, the laser beam travel 18 time from point to point. In third step, the laser beam travel 14 time from point to point. In fourth step, the laser beam travel 13 time from point to point.

\section{Conclusions}

Because of most machine learning or artificial intelligence problems, we are looking for the best model or strategy in all possible hypothesis spaces. The optimization problem can be divided into two sub-problems: how to construct a reasonable objective function and how to solve the objective function. We can see that some of the algorithms, we are familiar with can originally be regarded as some concrete the process of solving optimization problems.

Laser hardening can be integrated into the continuous production process. This quenching process is characterized by being environmentally friendly and clean. In addition, it opens up new possibilities for designers and production planners. For many years, it has been proved that laser quenching has obvious economic effects.

The ant and bee society is a well-organized 

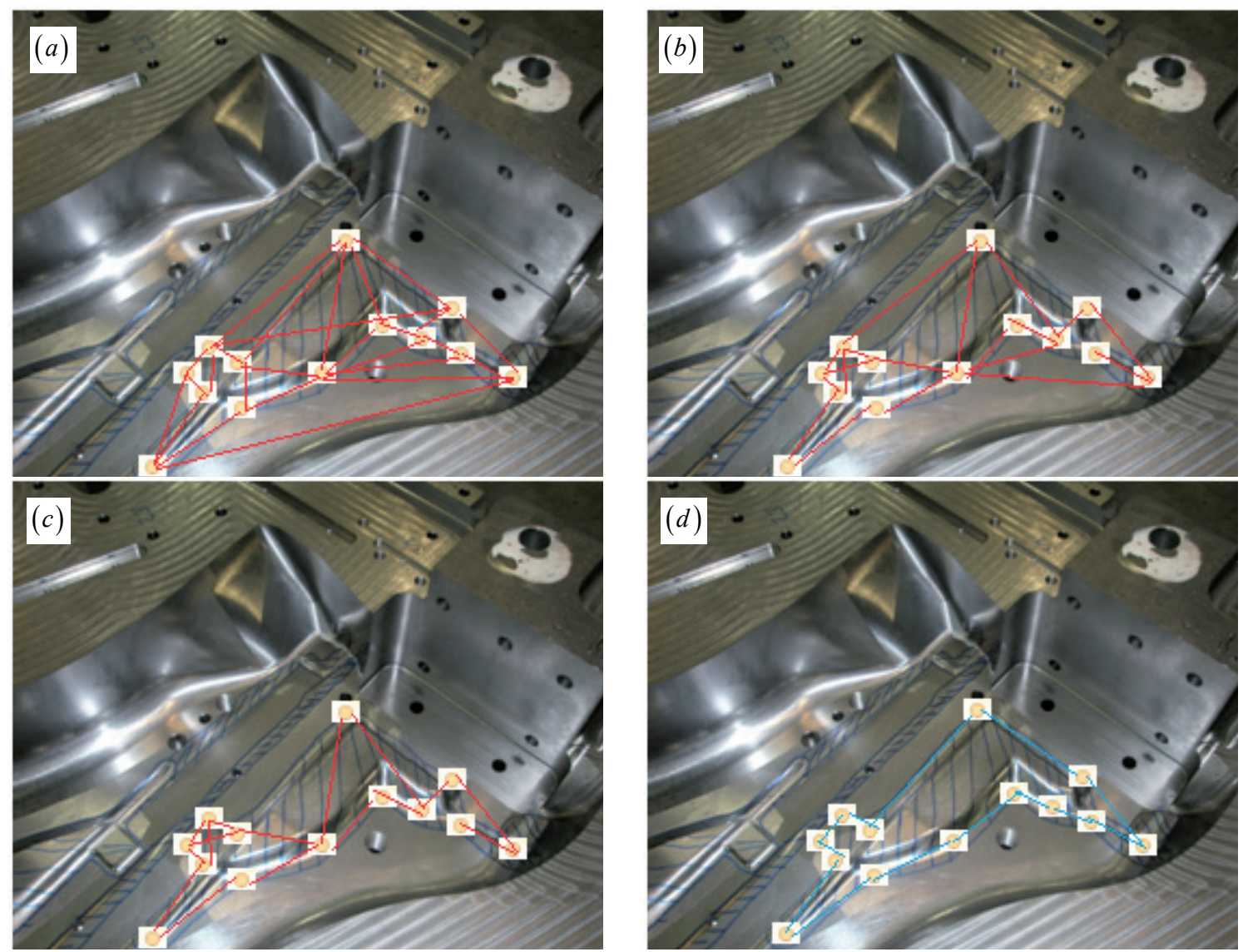

Figure 6: Different trajectory computed with hybrid algorithm: a)-c) examples of initial trajectories, d) final trajectory.

and well-structured structure with specific roles and standardized behaviors. So, we can use swarm intelligence to solve practical problems in engineering. In this article, is presented a new hybrid system of swarm intelligence using for minimal path travel on contours in mechanical engineering, especially in laser technology of hardening. It is presented application of swarm intelligence, ant colony and bee colony in optimization problem. It was shown using ant and bee colony to find optimal path of robot laser beam to harden material.

\section{References and Notes}

1. Piryonesi, S. M., Nasseri, M., Ramezani, A. (2018). Resource leveling in construction projects with activity splitting and resource constraints: a simulated annealing optimization. Canadian Journal of Civil Engineering. 46, 81-86.

2. Rosenberg, L., Lungren, M., Halabi, S., Willcox, G., Baltaxe, D., Lyons, M., (2018). Artificial Swarm Intelligence employed to Amplify Diagnostic Accuracy in Radiology. IEEE 9th
Annual Information Technology, Electronics and Mobile Communication Conference (IEMCON). Vancouver, BC: IEEE: 1186-1191. doi:10.1109/IEMCON.2018.8614883. ISBN 9781538672662.

3. Anukaran, K., Akhilesh, M., Vineet, T., Gupta, P.N. (2015). A literature based survey on swarm intelligence inspired optimization technique. International Journal of Advanced Technology in Engineering and Science Volume No 03, Special Issue No. 01

4. Yu, B., Chen, G. Swarm intelligence in mechanical engineering. Advances in Mechanical Engineering 2016, Vol. 8(12) 1-3. DOl: 10.1177/1687814016683595.

5. Babič, M. (2018) New double cycle hybrid method of machine learning using laser heat treatment pattern recognition with the topological properties of a network. Lasers in engineering, ISSN 0898-1507, vol. 40, no. 1/3, pp. 95-105

6. Fakir, R. Barka, N. Brousseau, J. (2018) Case study of laser hardening process applied to 4340 steel cylindrical specimens using simulation and experimental validation. Case Stud. Therm. Eng. 11, 15-25. 
Acta Mechanica Slovaca

Journal published by Faculty of Mechanical Engineering - Technical University of Košice

7. Grum, J. (2007) Comparison of different techniques of laser surface hardening. Journal of Achievements of Materials and Manufacturing Engineering. 24(1).

8. Abolmaali S., Roodposhti F.R. (2018). Portfolio Optimization Using Ant Colony Method a Case Study on Tehran Stock Exchange. Journal of Accounting. Mar, 8(1).

9. Madhumala R.B., Tiwari H. (2020) Analysis of Virtual Machine Placement and Optimization Using Swarm Intelligence Algorithms. In: Haldorai A., Ramu A., Khan S. (eds) Business Intelligence for Enterprise Internet of Things. EAl/Springer Innovations in Communication and Computing. Springer, Cham. https://doi.org/10.1007/978-3-030-44407-5_9.

10. Colorni, A., Dorigo, M., Maniezzo, V. (1991) Distributed Optimization by Ant Colonies. Appeared in proceedings of ecal91 - European conference on artificial life, Paris, France, Elsevier publishing, 134-142.

11. Kiran, M. S., Babalik, A. (2014) Improved Artificial Bee Colony Algorithm for Continuous Optimization Problems. Journal of Computer and Communications, 2, 108-116.

12. Karaboğa, D. (2005). An Idea Based on Honey Bee Swarm for Numerical Optimization. S2CID 8215393. 